\title{
RPL22 Gene
}

National Cancer Institute

\section{Source}

National Cancer Institute. RPL22 Gene. NCI Thesaurus. Code C97761.

This gene is involved in translation. 